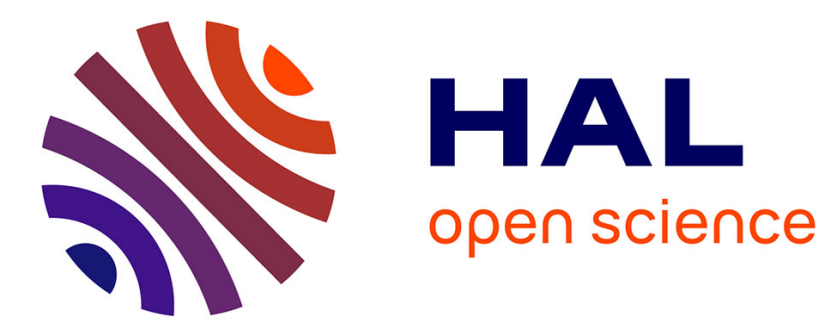

\title{
Longevity, Pollution and Growth
}

Natacha Raffin, Thomas Seegmuller

\section{To cite this version:}

Natacha Raffin, Thomas Seegmuller. Longevity, Pollution and Growth. 2012. halshs-00793513

\section{HAL Id: halshs-00793513 \\ https://shs.hal.science/halshs-00793513}

Preprint submitted on 22 Feb 2013

HAL is a multi-disciplinary open access archive for the deposit and dissemination of scientific research documents, whether they are published or not. The documents may come from teaching and research institutions in France or abroad, or from public or private research centers.
L'archive ouverte pluridisciplinaire HAL, est destinée au dépôt et à la diffusion de documents scientifiques de niveau recherche, publiés ou non, émanant des établissements d'enseignement et de recherche français ou étrangers, des laboratoires publics ou privés. 


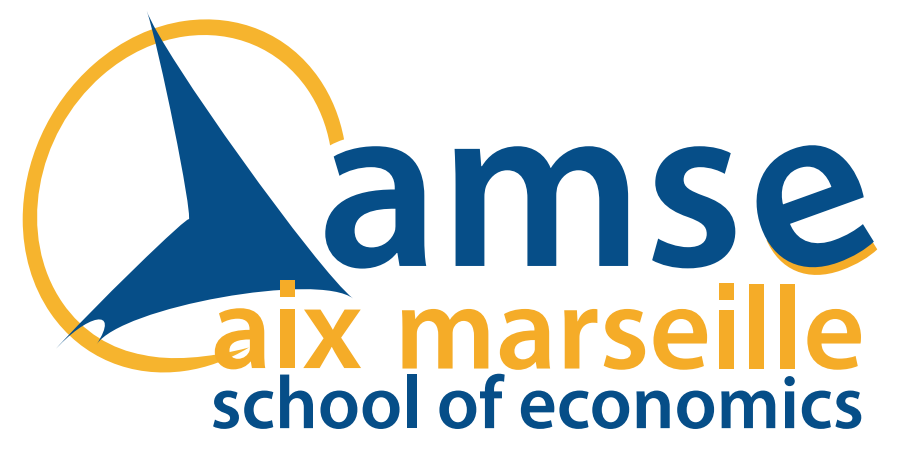

\section{Working Papers / Documents de travail}

Longevity, Pollution and Growth

Natacha Raffin

Thomas Seegmuller 


\title{
Longevity, pollution and growth*
}

\author{
Natacha Raffin ${ }^{\dagger}$ and Thomas Seegmuller ${ }^{\ddagger}$
}

October 30, 2012

\begin{abstract}
We analyze the interplay between longevity, pollution and growth. We develop an OLG model where longevity, pollution and growth are endogenous. The authorities may provide two types of public services, public health and environmental maintenance, that participate to increase agents' life expectancy and to sustain growth in the long term. We show that global dynamics might be featured by a high growth rate equilibrium, associated with longer life expectancy and a environmental poverty trap. We examine changes in public policies: increasing public intervention on health or environmental maintenance display opposite effects on global dynamics, i.e. on the size of the trap and on the level of the stable balanced growth path. On the contrary, each type of public policy induces a negative leverage on the long run rate of growth.
\end{abstract}

JEL classification: I15; O44; Q56;

Keywords: Life expectancy; Pollution; Health; Growth.

\section{Introduction}

As it is commonly acknowledged in the literature, there exists a complementarity between improvements in individuals' health status, especially life expectancy, and economic development. Healthier agents may be more productive at work, they are less absent and display more cognitive capacities, thus stimulating growth. Indeed, the reasoning is quite intuitive: an augmented health status induces agents to accumulate more capital (be it physical or human capital) since an increased longevity enhances the rate of return of investments (education, savings among others). Individuals can enjoy and benefit longer from their investment in the future. Hence, by promoting accumulation of assets, longevity has crucial

\footnotetext{
${ }^{*}$ We would like to thank C. Withagen for his helpful comments and all participants to the EAERE 2012 and EEA-ESEM 2012 conferences. This paper also benefits from the financial support of French National Research Agency Grant (ANR-09-BLAN-0350-01).

${ }^{\dagger}$ Corresponding author. University Paris Ouest Nanterre la Défense, EconomiX and Climate Economics Chair. Building G, office 604b, 200 av. de la République, 92001 Nanterre cedex, France. Tel: +33 140977781 . E-mail: nraffin@u-paris10.fr

łAix-Marseille University (Aix-Marseille School of Economics), CNRS and EHESS, Centre de la Vieille Charité, 2 rue de la Charité, 13236 Marseille CEDEX 02, France. E-mail: thomas.seegmuller@univ-amu.fr.
} 
repercussions on development in the long term. In turn, governments of richer countries are able to levy more resources to finance health structures, provide health services and improve access to the latter, while the economy develops. In the end, these two key ingredients feed on each other to promote growth. Theoretical papers like Blackburn and Cipriani (2002), Chakraborty (2004) or Cervelatti and Sunde (2005) also provide an illustration to such arguments. ${ }^{1}$

Unfortunately, economic growth might also be a source of negative externalities by deteriorating the environment. This damaging effect is not without consequences on health status, but it even may be a major cause of reduced longevity. Typically, the World Health Organization ${ }^{2}$ points out that outdoor pollution might be responsible worldwide for $9 \%$ of lung cancer deaths or $5 \%$ of cardiopulmonary deaths while $88 \%$ of diarrhoal deaths are attributable to unsafe water. Broadly speaking, climate change causes 150000 excess deaths annually while indoor pollution kills 2 millions people in 2012. This phenomena has often been addressed by economists and is also a major concern for many physicians (see among others Pope (2000), Chay and Greenstone (2003), Dasgupta (2004), Evans and Smith (2005), WHO (2004, 2006)). Most empirical studies usually conclude to a significant and detrimental impact of air (or/and water) pollution on health status, in particular those of children and elderly people. For instance, Chay and Greenstone (2003) show that a reduction in $1 \mathrm{mg} / \mathrm{m}^{3}$ in particulates results in 4-8 fewer infant deaths per 100000 live births. Even though the magnitude of these effects is still discussed, they argue that chronic as well as acute episodes of pollution can translate into pulmonary and cardio-vulnerability, neuropsychiatric disorders, more hospital admissions, diseases and even deaths attributable to environmental hazards.

Hence, regarding these two types of argument and evidence, changes in longevity should be considered with respect to both the evolution of environmental quality and the development of health policies. Thus, the authorities might intervene and undertake appropriate actions to reduce health impacts induced by a damaged environmental quality in order to promote growth and development. These actions could be of two distinct types: (i) a public health policy to weaken harmful effects of pollution on health status or/and (ii) a public environmental policy in order to reduce environmental damages.

The aim of this paper is precisely to study the dynamic path of pollution and economic growth when households' longevity is endogenously determined by both health policy and environmental quality. We are especially concerned with the effects of a public intervention, taking the form of health spendings as well as environmental expenses.

In our overlapping generations model, households live at most two periods, since the length of second period life is uncertain. As supported by the empirical literature, longevity is driven by two main factors: public health spending and environmental quality. Health services and environmental maintenance are financed thanks to taxes on labor income and provided by authorities. A priori, the two types of public expenditures seem to play a similar role by improving life expectancy. However, they differ in many ways so that the design of the public policy is a key issue of our analysis. Indeed, the flow of health expenditures could be interpreted as "curative" actions undertaken by the authorities to treat ill-health individuals and directly improve life expectancy. Differently, the environmental expenditures

1. See Mirowsky and Ross (1998) for a detailed survey on the relationship between human capital and longevity.

2. One can also refer for instance to the reports by the World Health organization on the indicator of environmental health. 
could be regarded as "preventive" actions engaged in order to diminish the future harmful stock of pollution, which captures the level of environmental quality within the economy. This stock grows through pollution flows that are a direct by-product of production meanwhile it is reduced thanks to cleaning activities provided by the authorities.

As a consequence of microeconomic behaviors, physical capital and pollution evolve jointly. Because we introduce an AK-type technology, both grow endogenously. Studying global dynamics and focusing on the design of the public intervention, we show that multiple steady states, associated with balanced growth paths, and the emergence of an environmental poverty trap are driven by the endogenous longevity but also the existence of pollution as a stock.

Furthermore, we argue that the two instruments exhibit different outcomes on global dynamics. When multiple equilibria exist, an augmentation of preventive environmental actions induces ambiguous effects. On the one hand, it enlarges the environmental poverty trap but, on the other hand, it rises the ratio of capital over pollution of the stable steady state, characterized by a larger growth rate. Therefore, a higher green fiscal pressure, for a given level of public health expenditures, is beneficial only to economies who have already reached a critical level of development. Otherwise, it could lead one economy into a vicious cycle and drives it to the environmental poverty trap. In contrast, when we examine the effects of a change in the level of curative actions, the results are opposite. Hence, a rise in the health tax rate is detrimental for the economies which have already reached a high enough level of economic development. Nonetheless, it can help one economy initially stuck into the environmental poverty trap to step out and reach a steady state with a larger growth rate in the long term. In any case, this analysis of global dynamics implies that the two types of public spending display different outcomes and are not substitutable.

However, this conclusion is no more relevant when we focus on the effects of health and environmental policies on the growth rates at a steady state. Indeed, an augmented level of either the environmental or health expenditures has a negative impact on economic growth.

We may note that in this paper, we focus on the effects of policy instruments on global dynamics, i.e. the size of the environmental poverty trap and the level of development in the long-run, and on the growth rate. However, in line with Bovenberg and Smulders (1996) or Ono (2003), we ignore welfare issues. Indeed, some policies, especially environmental ones, face informational and political constraints, and have difficulties to fully internalize externalities that prevent the government to correctly implement the optimal policy.

The paper is based on the well-known one by Chakraborty (2004). Indeed, we extend his framework to take into account pollution as an additional determinant of longevity. Jouvet et al. (2010) is also linked to our paper, but in contrast to us, dynamics are not examined. Moreover, our paper is close to Varvarigos (2009) and Palivos and Varvarigos (2012), as they consider that longevity is positively affected by public spending and negatively by pollution. But, different from them, we consider dynamics of the environmental variable since pollution is no longer a flow but a stock that depends on past pollution flows, production and public cleaning activities. Finally, in contrast to Balestra and Dottori (2012), we ignore the implications of aging on voting decision, but have growth.

The rest of this paper is organized as follows. In the next section, we present the model. In Section 3, we define the inter-temporal equilibrium. Steady states characterized by balanced growth paths are analyzed in Section 4 . Section 5 is devoted to the analysis of dynamics. The effects of policies on the level of growth are derived in Section 6, while some 
concluding remarks appear in Section 7. Proofs and technical details are relegated to the Appendix.

\section{The model}

In this section, we present our basic framework and derive the optimal microeconomic choices of agents.

\subsection{Production}

In this economy, one unique final good is produced by a continuum of competitive firms using the following technology: $Y_{t}=F\left(K_{t}, \bar{K}_{t} L_{t}\right)$, where $Y_{t}$ is aggregate production, $K_{t}$ aggregate capital, $L_{t}$ aggregate labor. The production function $F\left(K_{t}, \bar{K}_{t} L_{t}\right)$ has usual properties and is homogeneous of degree 1 . The variable $\bar{K}_{t}$ accounts for the aggregate (average) capital, which is taken as given by private firms. Hence, the production benefits from externalities (which are labour-augmenting) that will be the engine of endogenous growth.

Let us note $w_{t}$ the real wage and $r_{t}$ the real interest rate. Profits maximization by firms yields:

$$
\begin{aligned}
w_{t} & =F_{2}\left(K_{t} / \bar{K}_{t}, L_{t}\right) \bar{K}_{t} \\
r_{t} & =F_{1}\left(K_{t} / \bar{K}_{t}, L_{t}\right)
\end{aligned}
$$

where $F_{i}$ denotes the partial derivatives of function $F$ with respect to its $i t h$ argument.

\subsection{Households}

In this overlapping generations model, agents live for two periods. There is no population growth so that the population size of a young generation is normalized to one. Time is discrete and indexed by $t=0, \ldots,+\infty$. When young, an household lives during the entire period. He consumes the final good $\left(c_{t}\right)$ and saves $\left(s_{t}\right)$. When old, agents' time length ${ }^{3}$ is given by $\phi_{t} \leqslant 1$, namely the longevity. They also consume $d_{t+1}$ at each moment of time. The remunerated saving is used to finance this old consumption. The preferences of an household born at date $t$ are represented by the following utility function:

$$
U_{t}=\ln c_{t}+\phi_{t} \ln d_{t+1},
$$

where $\phi_{t}$ captures agents' longevity. In this paper, we emphasize the important role played by both health care expenses $\left(H_{t}\right)$ and the reduction of the pollution stock $\left(P_{t}\right)$ to improve agents' health status and, in particular, longevity. Indeed, we consider the net effect of pollution on health, taking into account the government expenditures as a mean to protect against pollution-induced damages. Formally, the critical determinant of life expectancy becomes the ratio $H_{t} / P_{t}$. This setup, crucial to our analysis, can be supported by a large body of both economic and medical empirical studies. Mainly microeconomic, those studies point out that air pollution is likely to have a greater impact on agents with lower access

3. The variable $\phi_{t}$ can also be interpreted as a survival probability. 
to primarily medical care (see Bates (1995)). Typically, in their study lead in New York City (US), Gwynn and Thurston (2001) assess the contribution of socioeconomic status (hereafter SES) to any observed racial differences in pollution effects. They show that discrepancies in air pollution effects among agents stem from health care disparities, in particular for Ozone $\left(\mathrm{O}_{3}\right)$ pollution. They calculate a relative risk for respiratory hospital admission and show that the latter belongs to the interval 1.032 to 1.12 according to the racial category. Similarly, Niedell (2004) in his study on Californian (US) data emphasize larger pollution exposure for low SES children. The author shows that a decrease in pollution level from 1992 to 1998 reduced asthma rates from 5-14 \% meanwhile children with low SES suffer greater harm from air pollution.

To be more specific, we assume that $\phi_{t} \equiv \phi\left(H_{t} / P_{t}\right)$, where $\phi^{\prime}() \geqslant .0 \geqslant \phi^{\prime \prime}($.$) and$ $0 \leqslant \phi(0)=\delta / \epsilon \leqslant \phi(+\infty)=b \leqslant 1$. Let us consider the following function that satisfies these properties:

$$
\phi\left(H_{t} / P_{t}\right)=\frac{\delta+b H_{t} / P_{t}}{\epsilon+H_{t} / P_{t}}
$$

with $\delta \geqslant 0$ and $\epsilon \geqslant 0$. Let us underline that when $\delta=\epsilon=0$, the longevity becomes constant, i.e. $\phi_{t}=b$. Taking now $\epsilon>0$, longevity is still positive despite the absence of health expenditures $\left(H_{t}=0\right)$ if $\delta>0$. Moreover, when $\delta$ increases from 0 to $\epsilon b$, longevity becomes less dependent on $H_{t} / P_{t}$ and less concave, for a given level of the ratio $H_{t} / P_{t}$. For tractability reasons, this functional form will be used all along the paper to derive main analytical results. ${ }^{4}$

The household maximizes the utility function (3) considering $\phi_{t}, w_{t}, R_{t+1}$, the fiscal policy as given and facing the two following budget constraints:

$$
\begin{aligned}
c_{t}+s_{t} & =\left(1-\tau_{1}-\tau_{2}\right) w_{t} \\
\phi_{t} d_{t+1} & =R_{t+1} s_{t},
\end{aligned}
$$

with $\tau_{1}$ the tax rate that finances public health expenditures and $\tau_{2}$ the one which allows for environmental maintenance ${ }^{5}$ and $R_{t+1}$ is the gross return on investment. Note that following Chakraborty (2004) or Varvarigos (2009) among the others, we assume a perfect annuity market. The return of total savings realized by the young is equally distributed among survival old households. Using (5) and (6), we can also define the life-cycle budget constraint:

$$
c_{t}+\frac{\phi_{t}}{R_{t+1}} d_{t+1}=\left(1-\tau_{1}-\tau_{2}\right) w_{t}
$$

Maximizing utility function (3) under the inter-temporal budget constraint (7) yields the

4. Note that Blackburn and Cipriani (2002) and Castelló-Climent and Doménech (2008) use closely related expressions for the longevity in their paper.

5. We could, of course, have considered that only one tax is levied on labour income and the product of this taxation shared among curative and preventive expenditures. For the clarity of the paper and the comparative statics exercise presented in the following section, we argue that such our set-up is more adapted. Let us notice that he two modeling choices are otherwise equivalent. 
optimal microeconomic choices of an household:

$$
\begin{aligned}
c_{t} & =\frac{\left(1-\tau_{1}-\tau_{2}\right) w_{t}}{1+\phi_{t}} \\
d_{t+1} & =\frac{R_{t+1}\left(1-\tau_{1}-\tau_{2}\right) w_{t}}{1+\phi_{t}}
\end{aligned}
$$

Using (6) and (9), we easily deduce individuals' savings:

$$
s_{t}=\frac{\phi_{t}\left(1-\tau_{1}-\tau_{2}\right) w_{t}}{1+\phi_{t}}
$$

Both consumption and savings are negatively affected by the fiscal policy through an income effect. Longevity tends to lower consumption during youth but stimulates savings, as in Chakraborty (2004).

\subsection{Public sector}

In our setup, the public sector provides health services $H_{t}$, which directly increase longevity, and public environmental spendings $G_{t}$, which help improving environmental quality (and so indirectly increase life expectancy). The curative health expenditures embody, among others, the infrastructures of the medical system, the access to health care, the medical technological level. The preventive actions gather all abatement activities engaged by public authorities that affect the stock of pollution. Both are financed by labor income taxation so that:

$$
\begin{aligned}
H_{t} & =\tau_{1} w_{t} L_{t} \\
G_{t} & =\tau_{2} w_{t} L_{t},
\end{aligned}
$$

with $\tau_{1}+\tau_{2}<1$. Equations (11) and (12) mean that government acts as if there are two separate balanced-budget constraints. In contrast to Varvarigos (2009) or Palivos and Varvarigos (2012), this will allow us to treat the two types of expenditures as independent.

\subsection{Environmental quality}

Finally, in this set-up, we introduce a variable that accounts for the deterioration of the environment: the stock of pollution. We assume that the future pollution stock depends on past pollution $P_{t} \geqslant 0$, like air or water pollution for instance. We also consider that it grows with production meanwhile it diminishes thanks to public cleaning activities. Thus, the law of motion of pollution can be written as:

$$
P_{t+1}=(1-m) P_{t}+a_{1} Y_{t}-a_{2} G_{t}
$$

with $m \in(0,1]$ the natural absorption of pollution, $a_{1} \geqslant 0, a_{2} \geqslant 0$, and $P_{0} \geqslant 0$ given. The stock of physical capital displays two opposite effects on the accumulation of pollution. On the one hand, production has a side-effect and generates pollution with an exogenous technology, $a_{1}$; On the other hand, production induces public receipts that finance cleaning activities with a given technology, $a_{2}$. 


\section{Equilibrium}

Being given that the population size of a young working generation equals one, the equilibrium in the labor market is ensured by $L_{t}=1$. At the equilibrium, we also have $\bar{K}_{t}=K_{t}$. Let us define $A \equiv F(1,1)$ and $\alpha \equiv F_{1}(1,1) / F(1,1)$, which implies that $1-\alpha=F_{2}(1,1) / F(1,1)$. Then, as in a standard AK model, equations (1) and (2) rewrite:

$$
\begin{aligned}
w_{t} & =(1-\alpha) A K_{t} \\
r_{t} & =\alpha A
\end{aligned}
$$

and production is given by $Y_{t}=A K_{t}$.

According to the law of motion of pollution and using equations (12) and (14), equation (13) rewrites:

$$
P_{t+1}=(1-m) P_{t}+\left(a_{1}-a_{2} \tau_{2}(1-\alpha)\right) A K_{t}
$$

In the following, we realistically assume that pollution flows are strictly positive:

Assumption 1. $a_{1}>a_{2} \tau_{2}(1-\alpha)$

Note that this parametric assumption enforces an additional upper bound only on the environmental tax rate $\tau_{2}$, and consequently on the level of preventive expenses. Such an assumption finds a support in the paper by Economides and Philippopoulos (2008), for instance.

In addition, equilibrium on the capital market is satisfied when $K_{t+1}=s_{t}$, that is savings are used to finance the capital stock. Using (10), this is equivalent to:

$$
K_{t+1}=\frac{\phi_{t}}{1+\phi_{t}}\left(1-\tau_{1}-\tau_{2}\right)(1-\alpha) A K_{t}
$$

Finally, longevity can be defined as a function of the ratio capital over pollution, $x_{t} \equiv$ $K_{t} / P_{t}$, at the equilibrium. ${ }^{6}$ Indeed, using (14), we can express the survival probability as follows:

$$
\phi_{t}=\phi\left(\tau_{1}(1-\alpha) A x_{t}\right)
$$

Substituting (18) in (17), the dynamics of the economy are, a priori, characterized by a two-dimensional system (16)-(17), $K_{0} \geqslant 0$ and $P_{0} \geqslant 0$ being given. This system describes the evolution of both pollution and capital over time. Since there exists an externality of physical capital, growth is endogenous and the variables $K_{t}$ and $P_{t}$ do not reach a stationary value in the long term. However, defining the capital growth factor $g_{k t} \equiv K_{t+1} / K_{t}$, equation (17) can be rewritten:

$$
g_{k t}=\frac{\phi\left(\tau_{1}(1-\alpha) A x_{t}\right)}{1+\phi\left(\tau_{1}(1-\alpha) A x_{t}\right)}\left(1-\tau_{1}-\tau_{2}\right)(1-\alpha) A
$$

Using (16) and (18), we get:

$$
x_{t+1}=\frac{\phi\left(\tau_{1}(1-\alpha) A x_{t}\right)}{1+\phi\left(\tau_{1}(1-\alpha) A x_{t}\right)} \frac{\left(1-\tau_{1}-\tau_{2}\right)(1-\alpha) A x_{t}}{1-m+\left(a_{1}-a_{2} \tau_{2}(1-\alpha)\right) A x_{t}}
$$

6. Note that this ratio is proportional to the inverse of the pollution intensity of production. 
We can observe that, given the path of the predetermined variable $x_{t}$, we determine the growth factor $g_{k t}$. Hence, analyzing the potential steady states and the dynamics of $x_{t}$, we deduce the balanced growth paths and their stability properties. More specifically, substituting (4) into (20), dynamics are driven by:

$$
x_{t+1}=\frac{\delta+b \tau_{1}(1-\alpha) A x_{t}}{(\epsilon+\delta)+(1+b) \tau_{1}(1-\alpha) A x_{t}} \frac{\left(1-\tau_{1}-\tau_{2}\right)(1-\alpha) A x_{t}}{1-m+\left(a_{1}-a_{2} \tau_{2}(1-\alpha)\right) A x_{t}} \equiv \psi\left(x_{t}\right)
$$

\section{Steady state analysis}

A steady state is a solution $x \geqslant 0$ such that $x_{t+1}=x_{t}=x=\psi(x)$. At a steady state, the rate of growth of physical capital is identical to the one of pollution stock. According to equation (21), it should satisfy:

$$
x=\frac{\delta+b \tau_{1}(1-\alpha) A x}{(\epsilon+\delta)+(1+b) \tau_{1}(1-\alpha) A x} \frac{\left(1-\tau_{1}-\tau_{2}\right)(1-\alpha) A x}{1-m+\left(a_{1}-a_{2} \tau_{2}(1-\alpha)\right) A x}
$$

A quick look at equation (22) allows us to claim that one steady state is obviously $x=0$. However, besides this trivial stationary value, other solutions $x>0$ might exist. These results are summarized in the following proposition:

Proposition 1. Let

$$
\begin{aligned}
& A_{0}(\delta) \equiv \frac{(\epsilon+\delta)(1-m)}{\delta\left(1-\tau_{1}-\tau_{2}\right)(1-\alpha)} \\
& A_{1}(\delta) \equiv \frac{(1-m)(1+b) \tau_{1}(1-\alpha)+(\epsilon+\delta)\left(a_{1}-a_{2} \tau_{2}(1-\alpha)\right)}{b \tau_{1}(1-\alpha)^{2}\left(1-\tau_{1}-\tau_{2}\right)}
\end{aligned}
$$

Under Assumption 1, $\epsilon b \geqslant \delta>0, \epsilon>0$ and $m<1$, the following generically holds:

1. If $A>A_{0}(\delta)$, there is a unique steady state $x_{0}>0$;

2. If $A<A_{0}(\delta)$, there exists a threshold value $\bar{\delta} \in(0, \epsilon b)$ such that $A_{0}(\delta)>A_{1}(\delta)$ for all $\delta<\bar{\delta}$. Then, for all $\delta<\bar{\delta}$ and $A \in\left(A_{1}(\delta), A_{0}(\delta)\right)$, with $A$ sufficiently close to $A_{0}(\delta)$, there exist two steady states $x_{1}(>0)$ and $x_{2}\left(>x_{1}\right)$.

Note that in the limit case where $m=1$, there is a unique steady state $x_{0}>0$, but the steady state $x=0$ is ruled out. When $\delta=\epsilon=0$, the steady state $x=0$ still remains and there is a unique steady state $x_{0}>0$ if and only if $A>\frac{(1-m)(1+b)}{\left(1-\tau_{1}-\tau_{2}\right)(1-\alpha) b}$.

Proof. See Appendix A.

Importantly, we should emphasize that the emergence of multiple equilibria is conditional to the existence of both an endogenous longevity $(\epsilon b \geqslant \delta>0)$ and inertia in the evolution of the pollution stock $(m<1)$. To that extent, we differ from the papers by Chakraborty (2004), Blackburn and Cipriani (2002), Varvarigos (2009) or Palivos and Varvarigos (2012). Indeed, from equation (20), we can see that the law of motion of $x_{t}$ (the stock 
of capital over pollution) is defined by the combination of two ratios. The first one corresponds to the saving rate $\phi_{t} /\left(1+\phi_{t}\right)$ and the second one to the ratio of net labor income over pollution. The first one is constant when $\delta=\epsilon=0$, whereas the second one is constant when $m=1$. As it is clear from the above analysis, the multiplicity of steady states $x_{1}$ and $x_{2}$ occurs if both these two effects play a role, that is longevity has to be endogenous and pollution to be less than proportional to income. Finally, note that, for $\epsilon>0$, this multiplicity is not ruled out when $\delta>0$, i.e. health services are not essential to have a nonzero longevity. However, $\delta$ has not to be too large, meaning that longevity should be sufficiently concave.

Using (19), we argue that to each stationary value $x$ corresponds a growth factor $g_{k}$, given by:

$$
g_{k}=\frac{\phi\left(\tau_{1}(1-\alpha) A x\right)}{1+\phi\left(\tau_{1}(1-\alpha) A x\right)}\left(1-\tau_{1}-\tau_{2}\right)(1-\alpha) A=1-m+\left(a_{1}-a_{2} \tau_{2}(1-\alpha)\right) A x
$$

where the last equality is obtained using (22). By direct inspection of (25), we deduce that $g_{k}$ is increasing in $x$, meaning that a steady state characterized by a larger level of capital per pollution is associated with a higher growth rate of capital. In particular, the growth rates can be ranked: $\left.g_{k}\right|_{x=x_{2}}>\left.g_{k}\right|_{x=x_{1}}$.

\section{Dynamics}

Let us now focus on the dynamic behavior of our economy, as we can obtain a full characterization of global dynamics.

Proposition 2. Under Assumption 1, $\epsilon b \geqslant \delta>0, \epsilon>0$ and $m<1$, the following generically holds:

1. For $A>A_{0}(\delta)$, the unique steady state $x_{0}>0$ is stable, while $x=0$ is unstable;

2. For all $\delta<\bar{\delta}$ and $A \in\left(A_{1}(\delta), A_{0}(\delta)\right)$, with $A$ sufficiently close to $A_{0}(\delta)$, the steady state $x_{1}$ is unstable, $x_{2}\left(>x_{1}\right)$ is stable, while $x=0$ is stable.

Note that in the limit case where $m=1$, the steady state $x_{0}>0$ is globally stable. When $\delta=\epsilon=0$, the steady state $x_{0}>0$ is stable, while $x=0$ is unstable.

Proof. See Appendix B.

As it is illustrated in Figure 1a, this proposition shows that if $A>A_{0}(\delta)$, the economy monotonically converges to $x_{0}>0$, no matter initial conditions. Conversely, as described in Figure 1b, if the level of productivity in the economy is not so high, then multiplicity of equilibria occurs. In that case, initial conditions determine the long run situation reached by the economy. Let us consider one economy initially located on the left-hand side of $x_{1}$. Then, $x_{t}$ is relegated to the environmental poverty trap. During the convergence phase, capital over pollution decreases and one is driven to a steady state with a zero stock of physical capital. The mechanisms at stake are quite intuitive: if initially, the stock of capital over pollution is low, so does longevity. Young agents are not likely to save but favor current consumption. Despite preventive expenditures, pollution increases more than physical 


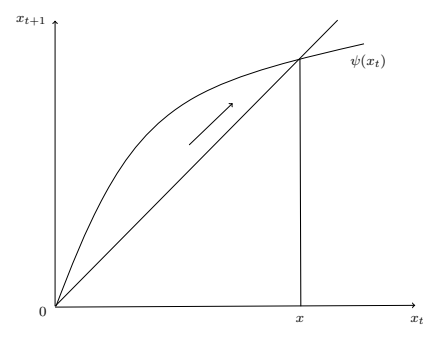

(a) $A>A_{0}(\delta)$

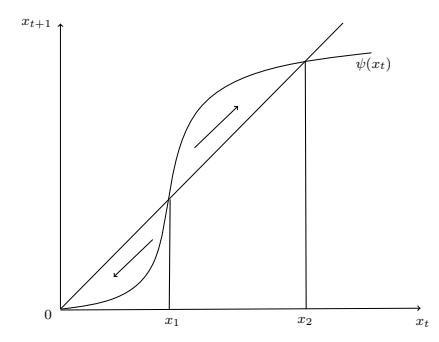

(b) $\delta<\bar{\delta}$ and $A \in\left(A_{1}(\delta), A_{0}(\delta)\right)$

Figure 1. Global Dynamics

capital. Consequently, it induces a reduction of life expectancy and leads the economy into a vicious cycle. In contrast, if the economy is initially located on the right-hand side of $x_{1}$, it converges monotonically to the high stable steady state, $x_{2}$. In that configuration, the ratio of capital over pollution always increases to reach its stationary value, meaning that the stock of capital rises more than pollution. At the steady state, physical capital and pollution grow at the same rate, and longevity is higher. In other words, if the initial stock of capital is sufficiently large regarding the initial stock of pollution, life expectancy is long enough to foster savings, since curative health services are sufficiently large with respect to pollution.

\subsection{The effects of preventive environmental expenditures on dynamics}

Let us now investigate more deeply the effect of abatement activities on the dynamics. We focus on the most interesting case where there is an environmental poverty trap (Proposition 
2.2). The results concerning case 1 of Proposition 2 can be easily deduced from the analysis below.

To evaluate all the potential effects at stake on the dynamics, we start by analyzing how $\psi\left(x_{t}\right)$, as defined in equation (21), evolves according to an increase in $\tau_{2}$. Using

$$
\frac{\partial \psi(x)}{\partial \tau_{2}}=(1-\alpha) A x \frac{\delta+b \tau_{1}(1-\alpha) A x}{\epsilon+\delta+(1+b) \tau_{1}(1-\alpha) A x} \frac{A x\left(a_{2}(1-\alpha)\left(1-\tau_{1}\right)-a_{1}\right)-(1-m)}{\left[1-m+\left(a_{1}-a_{2} \tau_{2}(1-\alpha)\right) A x\right]^{2}}
$$

We can deduce the following lemma:

Lemma 1. Let

$$
x_{a}=\frac{1-m}{A\left[a_{2}\left(1-\tau_{1}\right)(1-\alpha)-a_{1}\right]}
$$

Under Assumption 1, $\epsilon b \geqslant \delta>0, \epsilon>0$ and $m<1$, the following generically holds for all $\delta<\bar{\delta}$ and $A \in\left(A_{1}(\delta), A_{0}(\delta)\right)$, with $A$ sufficiently close to $A_{0}(\delta)$.

1. If $a_{1} \geqslant a_{2}\left(1-\tau_{1}\right)(1-\alpha), \partial \psi\left(x_{t}\right) / \partial \tau_{2}<0$ for all $x_{t}>0$. See Figure $2 a$.

2. If $a_{1}<a_{2}\left(1-\tau_{1}\right)(1-\alpha), \partial \psi\left(x_{t}\right) / \partial \tau_{2}<0$ for all $x_{t}<x_{a}$ and $\partial \psi\left(x_{t}\right) / \partial \tau_{2}>0$ for all $x_{t}>x_{a}$. See Figure $2 b$ drawn for $x_{1}<x_{a}<x_{2}$ and Figure $2 c$ for $x_{1}>x_{a}$.

From equation (21), we can easily observe the twofold effects of an increase in preventive actions on the ratio capital-pollution, $x_{t}$. On the one hand, there exists a negative income effect: Larger environmental expenditures reduce the remaining income to save and thus physical capital accumulation slows down. On the other hand, it displays a positive effect by reducing pollution flow. Since we have identified these two main mechanisms, we can state:

Proposition 3. Under Assumption 1, $\epsilon b \geqslant \delta>0, \epsilon>0$ and $m<1$, the following generically holds for all $\delta<\bar{\delta}$ and $A \in\left(A_{1}(\delta), A_{0}(\delta)\right)$, with $A$ sufficiently close to $A_{0}(\delta)$.

1. If $a_{1} \geqslant a_{2}\left(1-\tau_{1}\right)(1-\alpha)$, an increase of $\tau_{2}$ reduces the level of capital over pollution at the stable steady state, but enlarges the environmental poverty trap.

2. If $a_{1}<a_{2}\left(1-\tau_{1}\right)(1-\alpha)$, and if

a $x_{a}>x_{2}$, the effect of $\tau_{2}$ is similar to the previous case;

$b x_{1}<x_{a}<x_{2}$, an increase of $\tau_{2}$ increases the level of capital over pollution at the stable steady state, but also enlarges the environmental poverty trap;

$c x_{a}<x_{1}$, a rise of $\tau_{2}$ increases the level of capital over pollution at the stable steady state, but reduces the environmental poverty trap.

In configuration 1, the income effect always dominates, which explains that, following a rise in the level of preventive actions, one economy is more likely to fall into the trap. In addition, it could be the case that an economy, close on the right-hand side of $x_{1}$, that would have monotonically converge to the high steady state is now driven into the trap. Indeed, since $a_{1}$ is relatively high, the production technology is highly polluting and the maintenance technology is relatively less efficient. In that case, augmenting the amount of 


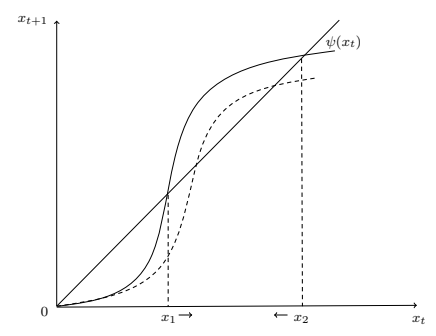

(a) when $a_{1}>a_{2}\left(1-\tau_{1}\right)(1-\alpha)$

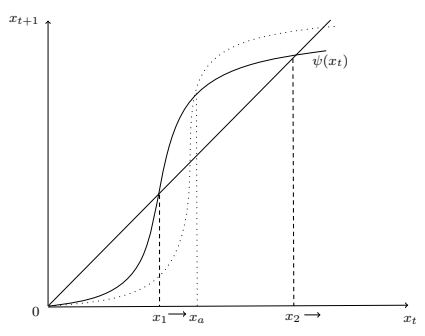

(b) when $a_{1}<a_{2}\left(1-\tau_{1}\right)(1-\alpha)$ and $x_{1}<$ $x_{a}<x_{2}$

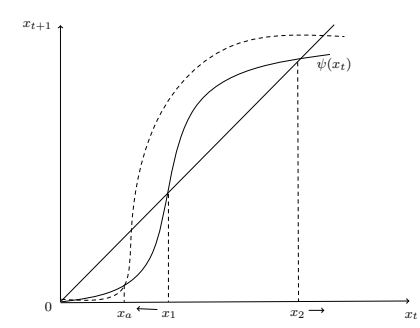

(c) when $a_{1}<a_{2}\left(1-\tau_{1}\right)(1-\alpha)$ and $x_{a}<x_{1}$

Figure 2 . An increase in preventive actions 
resources devoted to environmental maintenance first impedes the accumulation of physical capital but, in addition, is not sufficient to offset the harmful effect of production on the environment. Consequently, it can clearly slow down the economic development.

Conversely, in configuration 2, investing in preventive actions might be more appropriate. In particular, a more ambitious environmental public policy may exhibit a global positive effect: If the threshold value $x_{a}$ is sufficiently low, the economy reaches a higher steady state value and the development-environment trap is reduced (see configuration 2.c). Otherwise said, the reduced-pollution effect always dominates the income effect. For instance, one economy initially located close on the left of $x_{1}$, that would have been stuck into the trap, might be driven to the high equilibrium. This configuration is interesting with respect to the famous Environmental Kuznets Curve, pointed up by Grossman and Krueger (1995). The EKC pattern of development could be summarized as follows: For low stages of development, the economy develops while polluting and deteriorating the environment. Once a sufficiently high level of development/income is reached, the relationship inverts : The economy still develops but reduces pollution and thus improves environmental quality. Typically, one economy initially located close on the left-hand side of $x_{1}$ could experience such a non-monotonic pattern of development. First, since longevity is low, the economy collapses, but, following an increase in the public abatement activities, the ratio capital over pollution stops reducing but starts increasing to reach the high stationary equilibrium.

When $x_{a}$ belongs to the interval $\left[x_{1}, x_{2}\right]$, the results are more ambiguous. Even if the level of $x_{a}$ depends in a quite complicated manner on the parameter values, one can show that for $A$ sufficiently large, we have $\theta\left(x_{a}\right)<0$, that is $x_{1}<x_{a}<x_{2}$. As claimed in the corollary below, this means that this configuration is probably the most relevant one when $a_{1}<a_{2}\left(1-\tau_{1}\right)(1-\alpha)$.

Corollary 1. Let

$$
A_{\tau_{2}} \equiv \frac{(1-m) a_{2}\left[(1-m)(1+b) \tau_{1}(1-\alpha)+(\epsilon+\delta)\left(a_{2}\left(1-\tau_{1}\right)(1-\alpha)-a_{1}\right)\right]}{\left[a_{2}\left(1-\tau_{1}\right)(1-\alpha)-a_{1}\right]\left[(1-\alpha) b(1-m) \tau_{1}+\delta\left(a_{2}\left(1-\tau_{1}\right)(1-\alpha)-a_{1}\right)\right]}
$$

Under Assumption 1, $\epsilon b \geqslant \delta>0, \epsilon>0, m<1$ and $a_{1}<a_{2}\left(1-\tau_{1}\right)(1-\alpha)$, there exists

$\widetilde{\delta}>0$ such that $A_{\tau_{2}}<A_{0}(\delta)$ for all $\delta \in(0, \widetilde{\delta})$. Then, for all $\delta \in(0, \min \{\widetilde{\delta}, \bar{\delta}\})$ and $A \in$ $\left(\max \left\{A_{\tau_{2}}, A_{1}(\delta)\right\}, A_{0}(\delta)\right)$, with $A$ sufficiently close to $A_{0}(\delta)$, we have $x_{1}<x_{a}<x_{2}$, i.e. case 2.b of Proposition 3 applies.

Proof. See Appendix C.

On one side, augmented preventive actions lead the economy to reach a higher steady state value. But on the other side, the environmental poverty trap enlarges, so that one economy is more likely to converge to the low stable equilibrium $x=0$. For instance, an economy, that would have converged towards the high stationary value $x_{2}$, might be driven into the trap.

\subsection{The effects of curative health expenditures on dynamics}

Let us now isolate the role played by a more generous health policy, the level of expenditures devoted to abatement activities being constant. As above, we focus on the most inter- 
esting case 1.2 of (Proposition 2) and we note that results concerning case 1 of Proposition 2 can be easily deduced from the analysis below.

From (21), we can easily observe that an increase in the level of curative actions engaged by the authorities displays at least also two opposite effects on $x_{t}$ : (i) First, through a usual income effect, it reduces the revenue to be saved and thus has a negative effect on $x$; But (ii), second, it participates to augment longevity, thus fostering savings. The global effect depends on which effect dominates. We have:

$$
\frac{\partial \psi(x)}{\partial \tau_{1}}=\frac{(1-\alpha) A x}{\left[\epsilon+\delta+(1+b) \tau_{1}(1-\alpha) A x\right]^{2}\left[1-m+\left(a_{1}-a_{2} \tau_{2}(1-\alpha)\right) A x\right]} H(x)
$$

with

$H(x) \equiv-(\epsilon+\delta) \delta+A(1-\alpha)\left[b \epsilon\left(1-\tau_{2}-2 \tau_{1}\right)-\delta\left(1-\tau_{2}+2 b \tau_{1}\right)\right] x-b(1+b) \tau_{1}^{2}(1-\alpha)^{2} A^{2} x^{2}$

In the following lemma, we explain how $\psi(x)$ evolves according to a rise in curative health expenditures:

Lemma 2. Under Assumption 1, $\epsilon b \geqslant \delta, \epsilon>0$ and $m<1$, the following generically holds for all $\delta<\bar{\delta}$ and $A \in\left(A_{1}(\delta), A_{0}(\delta)\right)$, with $A$ sufficiently close to $A_{0}(\delta)$. There exists $\delta_{H} \in(0, \epsilon b)$ such that: ${ }^{7}$

1. If $\delta>\delta_{H}, \partial \psi\left(x_{t}\right) / \partial \tau_{1}<0$ for all $x_{t}>0$.

2. If $0 \leqslant \delta<\delta_{H}$, there exist $x_{1}^{H} \geqslant 0$ and $x_{2}^{H}\left(>x_{1}^{H}\right)$ such that $\partial \psi\left(x_{t}\right) / \partial \tau_{1}<0$ for all $x_{t}<x_{1}^{H}$, $\partial \psi\left(x_{t}\right) / \partial \tau_{1}>0$ for all $x_{1}^{H}<x_{t}<x_{2}^{H}$ and $\partial \psi\left(x_{t}\right) / \partial \tau_{1}<0$ for all $x_{t}>x_{2}^{H}$. Note that in the limit case where $\delta=0$, we have $x_{1}^{H}=0$.

Proof. See Appendix D.

Using Lemma 2, we can then prove the following (see Figure 3):

Proposition 4. Under Assumption 1, $\epsilon b \geqslant \delta=0, \epsilon>0$ and $m<1$, the following generically holds for all $\delta<\bar{\delta}$ and $A \in\left(A_{1}(\delta), A_{0}(\delta)\right)$, with $A$ sufficiently close to $A_{0}(\delta)$.

1. If $\delta>\delta_{H}$ or $A$ is sufficiently large and $\delta$ sufficiently low (in accordance with $A<A_{0}(\delta)$ ), an increase of $\tau_{1}$ reduces the level of capital over pollution at the stable steady state.

2. If $A$ is sufficiently large and $\delta$ sufficiently low (in accordance with $A<A_{0}(\delta)$ ), an increase of $\tau_{1}$ reduces the level of the environmental poverty trap.

Proof. See Appendix E.

This proposition shows that a policy promoting curative health care may have ambiguous effects. One economy initially located close on the left-hand side of $x_{1}$ that would have converged into the environmental poverty trap may escape from it by investing in curative health expenditures and finally reach the high stationary equilibrium. The augmented length of second period life time thanks to additional health expenditures allows to boost

7. The expressions of $x_{1}^{H}$ and $x_{2}^{H}$ are given in Appendix D. 


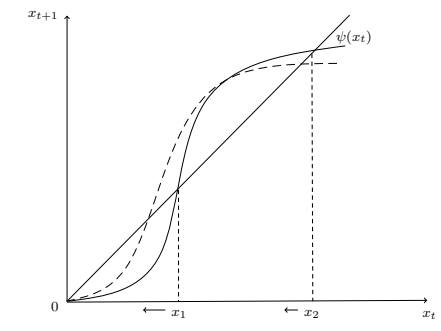

Figure 3. A rise in curative health expenditures

savings. Again, we are able to reproduce a non-monotonic development pattern like EKC. Nonetheless, if the economy is initially located on the right-hand side of $x_{1}$, the larger receipts devoted to health do not modify the development trajectory except that the equilibrium reached is lower. This negative impact is due to the properties of the longevity function. Indeed, life expectancy is increasing and concave with the ratio of capital over pollution. Then, as soon as $x_{t}$ increases, so does longevity, but less and less. Otherwise said, if the contribution to curative actions increases, but longevity is already high, the benefits on longevity are relatively low compared to the negative income effect. This also explains why a sufficiently low $\delta$ is required to have a reduced environmental poverty trap. Indeed, as seen above, the longevity is sufficiently concave and strongly depends on health services in this case. Therefore, the effect on longevity dominates the decrease of net income, taking into account of course that the ratio of capital-pollution is not too large.

Let us now concentrate on the policy recommendation we could draw from this above analysis, following Proposition 3, Corollary 1 and Proposition 4. We can show that the efficiency of each type of public expenditures crucially depends on the considered stage of development. Typically, in order to step out from the trap and for initial poor economies, it seems more appropriate to invest first in curative actions, that is to cure people that may suffer from bad hygienic conditions and deteriorated environment, the level of abatement activities being given. In a second step only, once the income reached by one economy and the longevity are high enough, it becomes more attractive to invest in preventive actions to sustain a higher level of capital compared to pollution.

\section{Growth, environmental and health policies}

In the previous section, we have underlined the different effects of each policy on dynamics. In this section, we are interested in their effects on the long-run rate of growth $\left(g_{k}\right)$. Focusing on a stable steady state, the results are summarized in the following proposition: 
Proposition 5. Under the assumptions of Propositions 3 and 4, the following holds at a Balanced Growth Path:

1. an increase of $\tau_{1}$ implies a decrease of $g_{k}$;

2. an increase of $\tau_{2}$ implies a decrease of $g_{k}$ if $\delta$ is sufficiently low and A sufficiently close to $A_{0}(\delta)$, in any case larger than $\frac{a_{2}(\epsilon+\delta)}{(1-\alpha) b \tau_{1}}$.

Proof. See Appendix F.

This result can be understood as follows. At a steady state which characterizes a balanced growth path, capital and pollution grow at the same rate.

Examining equation (25), we immediately see that the effect of curative health expenditures on growth only goes through the ratio of capital over pollution $x$. Yet, as seen in Proposition 4, $x$ is decreasing in $\tau_{1}$ at a stable steady state. This lowers pollution flow, and therefore the pollution growth. This induces a lower capital growth rate at a balanced growth path.

Focusing now on preventive environmental activities, we argue that the effect of $\tau_{2}$ goes through two channels on pollution flow. There is a direct one which always reduces pollution, (and so the growth rate) because it increases government expenditures devoted to improve environmental quality. An indirect one goes through the ratio capital over pollution, as before. When $x$ is decreasing in $\tau_{2}$, we can infer that the growth rates of pollution, and therefore of capital reduce. This occurs in cases 1 and 2.a of Proposition 3. In contrast, in cases 2.b and 2.c of Proposition 3, the direct and indirect effects go in opposite directions. As shown in Proposition 5, the direct effect dominates. A larger environmental tax rate induces a higher level of public spending, reducing the pollution growth rate as well as the capital one.

In the previous section, we have seen that environmental and health policies have opposite effects on the dynamics, enlarging or not the environment-development trap and increasing or not the level of capital over pollution at the stable steady state. On the contrary, focusing on the growth rates, we rather have to conclude that both health and environmental public expenditures exhibit similar effects. Even if surely our model is not sufficiently complicated to give some clear-cut policy recommendations, it asks the question on whether improving growth should be or not a relevant objective for the government.

\section{Conclusion}

We have analyzed the relationship between longevity, pollution and growth. We have presented an OLG model where growth, pollution and longevity are endogenous. Government intervention is twofold, providing health services and environmental maintenance. The analysis of global dynamics allows us to exhibit the coexistence of a stable balanced growth path with an environmental poverty trap. We stress out that the two public policies have opposite effects on dynamics, i.e. the size of the trap and the level of the stable steady state with a high level of growth, despite the fact that both raise longevity. On the contrary, increasing the contribution of either health services or environmental maintenance is detrimental for economic growth. Hence, focusing on positive issues of policy, recommendations are quite contrasted depending on the objective of the government. 


\section{Appendix}

\section{A Proof of Proposition 1}

A steady state with $x>0$ is a solution of the following equation:

$$
\begin{aligned}
\theta(x) \equiv & (1+b) \tau_{1}(1-\alpha) A^{2}\left(a_{1}-a_{2} \tau_{2}(1-\alpha)\right) x^{2}+\left[(1-m)(1+b) \tau_{1}(1-\alpha) A\right. \\
& \left.+\left(a_{1}-a_{2} \tau_{2}(1-\alpha)\right) A(\epsilon+\delta)-\left(1-\tau_{1}-\tau_{2}\right)(1-\alpha)^{2} A^{2} b \tau_{1}\right] x \\
& +(1-m)(\epsilon+\delta)-\delta\left(1-\tau_{1}-\tau_{2}\right)(1-\alpha) A=0
\end{aligned}
$$

Under Assumption 1, we have $\theta^{\prime \prime}(x)>0$ and $\theta(+\infty)=+\infty$. Two main cases emerge.

If $A>A_{0}(\delta)$, we get $\theta(0)<0$, which ensures that there exists a unique steady state, namely $x_{0}>0$.

If $A<A_{0}(\delta)$, we have $\theta(0)>0$. In this case, there is generically either no steady state, or two steady states. Let $\widetilde{x}$ such that $\theta^{\prime}(\widetilde{x})=0$. The existence of two steady states $x_{1}(>0)$ and $x_{2}\left(>x_{1}\right)$ is ensured by $\widetilde{x}>0$ and $\theta(\widetilde{x})<0$.

Using (A.1), we get:

$$
\tilde{x}=\frac{\left(1-\tau_{1}-\tau_{2}\right)(1-\alpha)^{2} A b \tau_{1}-(1-m)(1+b) \tau_{1}(1-\alpha)-\left(a_{1}-a_{2} \tau_{2}(1-\alpha)\right)(\epsilon+\delta)}{2(1+b) \tau_{1}(1-\alpha) A\left(a_{1}-a_{2} \tau_{2}(1-\alpha)\right)}
$$

Since the denominator is strictly positive, $\widetilde{x}>0$ requires $A>A_{1}(\delta)$.

We note that $A_{0}(\delta)$ is decreasing from $+\infty$ to $A_{0}(\epsilon b)$, when $\delta$ increases from 0 to its upper bound $\epsilon b$. In contrast, $A_{1}(\delta)$ is increasing from $A_{1}(0)$ to $A_{1}(\epsilon b)$, keeping finite values. Since $A_{1}(\epsilon b)>A_{0}(\epsilon b)$ but $A_{1}(0)<A_{0}(0)$, there exists $\bar{\delta} \in(0, \epsilon b)$ such that $A_{1}(\delta)<A_{0}(\delta)$ for all $\delta<\bar{\delta}$. In this case, the interval $\left(A_{1}(\delta), A_{0}(\delta)\right)$ is non-empty.

Now, substituting (A.2) in (A.1), we can show that $\theta(\widetilde{x})<0$ is equivalent to:

$$
\begin{aligned}
& {\left[\left(1-\tau_{1}-\tau_{2}\right)(1-\alpha)^{2} A b \tau_{1}-(1-m)(1+b) \tau_{1}(1-\alpha)-\left(a_{1}-a_{2} \tau_{2}(1-\alpha)\right)(\epsilon+\delta)\right]^{2}} \\
& >4(1+b) \tau_{1}(1-\alpha)\left(a_{1}-a_{2} \tau_{2}(1-\alpha)\right)\left[(\epsilon+\delta)(1-m)-\delta\left(1-\tau_{1}-\tau_{2}\right)(1-\alpha) A\right](\mathrm{A} .3)
\end{aligned}
$$

This inequality is satisfied if one takes $A$ sufficiently close to $A_{0}(\delta)$. Indeed, this ensures that the right-hand side of inequality (A.3) is arbitrarily weak, while the left-hand side is strictly positive.

Note that when $m=1, A_{0}(\delta)$ tends to 0 . The first case applies, showing that there is a unique steady state $x_{0}>0$. However, by direct inspection of (22) with $m=1, x=0$ is not a steady state. When $\delta=\epsilon=0$, we deduce that using (A.1), that there is one stationary solution $x_{0}>0$ if and only if $A>\frac{(1-m)(1+b)}{\left(1-\tau_{1}-\tau_{2}\right)(1-\alpha) b}$. Moreover, the steady state $x=0$ is not ruled out. This concludes the proof. 


\section{B Proof of Proposition 2}

The dynamics are driven by equation (21). We have $\psi(+\infty)=\frac{b(1-\alpha)\left(1-\tau_{1}-\tau_{2}\right)}{(1+b)\left(a_{1}-a_{2} \tau_{2}(1-\alpha)\right)}>0$ and $\psi(0)=0$. Moreover,

$$
\begin{aligned}
\psi^{\prime}(x)= & \frac{(1-\alpha) A\left(1-\tau_{1}-\tau_{2}\right)}{\Gamma(x)^{2}} \\
& {\left[\tau_{1}(1-\alpha) A(b \epsilon-\delta) x\left[1-m+\left(a_{1}-a_{2} \tau_{2}(1-\alpha)\right) A x\right]\right.} \\
& \left.+(1-m)\left(\delta+b \tau_{1}(1-\alpha) A x\right)\left[\epsilon+\delta+(1+b) \tau_{1}(1-\alpha) A x\right]\right]
\end{aligned}
$$

where $\Gamma(x) \equiv\left[\epsilon+\delta+(1+b) \tau_{1}(1-\alpha) A x\right]\left[1-m+\left(a_{1}-a_{2} \tau_{2}(1-\alpha)\right) A x\right]$ corresponds to the denominator of $\psi(x)$. We deduce that $\psi^{\prime}(+\infty)=0$ and

$$
\psi^{\prime}(0)=\frac{\left(1-\tau_{1}-\tau_{2}\right)(1-\alpha) \delta A}{(1-m)(\epsilon+\delta)}
$$

Therefore, $\psi^{\prime}(0)>1$ if $A>A_{0}(\delta)$, while $\psi^{\prime}(0)<1$ if $A<A_{0}(\delta)$. Using Proposition 1 and the continuity of $\psi(x)$, the results follow.

When $m=1$, we have:

$$
\psi\left(x_{t}\right)=\frac{\delta+b \tau_{1}(1-\alpha) A x_{t}}{(\epsilon+\delta)+(1+b) \tau_{1}(1-\alpha) A x_{t}} \frac{\left(1-\tau_{1}-\tau_{2}\right)(1-\alpha)}{a_{1}-a_{2} \tau_{2}(1-\alpha)}
$$

while when $\epsilon=\delta=0$,

$$
\psi\left(x_{t}\right)=\frac{b}{1+b} \frac{\left(1-\tau_{1}-\tau_{2}\right)(1-\alpha) A x_{t}}{1-m+\left(a_{1}-a_{2} \tau_{2}(1-\alpha)\right) A x_{t}}
$$

Using these two expressions of $\psi\left(x_{t}\right)$, we can easily deduce the stability properties when $m=1$ and $\epsilon=\delta=0$.

\section{Proof of Corollary 1}

Assuming that $a_{1}<a_{2}\left(1-\tau_{1}\right)(1-\alpha)$, we will show that under mild assumptions, case 2.b of Proposition 3 applies, or equivalently $\bar{x}_{1}<x_{a}<\bar{x}_{2}$. To do that, we have to show that $\theta\left(x_{a}\right)<0$, where $\theta(x)$ is defined in (A.1). Substituting (26) in (A.1), we obtain after some computations, that $\theta\left(x_{a}\right)<0$ is equivalent to $A>A_{\tau_{2}}$. Multiplicity of steady states requires $A<A_{0}(\delta)$. Therefore, we have to ensure the existence of a non-empty interval for $A$, i.e. $A_{\tau_{2}}<A_{0}(\delta)$. This last inequality is equivalent to:

$$
\begin{aligned}
& (1-m)\left(a_{2}\left(1-\tau_{1}\right)(1-\alpha)-a_{1}\right) \epsilon(1-\alpha) b \tau_{1}+\delta\left[\tau _ { 1 } ( 1 - \alpha ) ( 1 - m ) \left(b\left(a_{2} \tau_{2}(1-\alpha)-a_{1}\right)\right.\right. \\
& \left.\left.-a_{2}(1-\alpha)\left(1-\tau_{1}-\tau_{2}\right)\right)+\epsilon\left(a_{2}\left(1-\tau_{1}\right)(1-\alpha)-a_{1}\right)\left(a_{2} \tau_{2}(1-\alpha)-a_{1}\right)\right] \\
& +\delta^{2}\left(a_{2}\left(1-\tau_{1}\right)(1-\alpha)-a_{1}\right)\left(a_{2} \tau_{2}(1-\alpha)-a_{1}\right)>0
\end{aligned}
$$

Under Assumption 1 and $a_{1}<a_{2}\left(1-\tau_{1}\right)(1-\alpha)$, we immediately see that there is an upper bound $\widetilde{\delta}>0$ such that this inequality is satisfied if and only if $0<\delta<\widetilde{\delta}$. The corollary follows taking into account the conditions for the multiplicity of steady states. 


\section{Proof of Lemma 2}

Using (29), we see that for all $\delta \geqslant b \epsilon \frac{1-\tau_{2}-2 \tau_{1}}{1-\tau_{2}+2 b \tau_{1}}, H(x)<0$ for all $x>0$.

For $0 \leqslant \delta<b \epsilon \frac{1-\tau_{2}-2 \tau_{1}}{1-\tau_{2}+2 b \tau_{1}}$, we compute the discriminant $\Delta(\delta)$ of the polynomial of degree 2, $H(x)$. We obtain:

$$
\Delta(\delta)=A^{2}(1-\alpha)^{2}\left[b \epsilon\left(1-\tau_{2}-2 \tau_{1}\right)-\delta\left(1-\tau_{2}+2 b \tau_{1}\right)\right]^{2}-4 b(1+b) \tau_{1}^{2}(1-\alpha)^{2} A^{2}(\epsilon+\delta) \delta
$$

We deduce that $\Delta(0)>0$, while $\Delta\left(b \epsilon \frac{1-\tau_{2}-2 \tau_{1}}{1-\tau_{2}+2 b \tau_{1}}\right)<0$. Since $\Delta(\delta)$ is a polynomial of degree 2 in $\delta$, there is a unique $\delta^{H} \in\left(0, b \in \frac{1-\tau_{2}-2 \tau_{1}}{1-\tau_{2}+2 b \tau_{1}}\right)$, such that:

1. $\Delta(\delta)<0$ for all $\delta \in\left(\delta^{H}, b \in \frac{1-\tau_{2}-2 \tau_{1}}{1-\tau_{2}+2 b \tau_{1}}\right)$;

2. $\Delta(\delta)>0$ for all $\delta \in\left(0, \delta^{H}\right)$.

In case 1 , since $H^{\prime \prime}(x)<0$, we deduce that $H(x)<0$ for all $x>0$. In case $2, H(x)=0$ has two roots, $x_{1}^{H}$ and $x_{2}^{H}$, given by:

$$
\begin{aligned}
& x_{1}^{H}=\frac{A(1-\alpha)\left[b \epsilon\left(1-\tau_{2}-2 \tau_{1}\right)-\delta\left(1-\tau_{2}+2 b \tau_{1}\right)\right]-\sqrt{\Delta(\delta)}}{2 b(1+b) \tau_{1}^{2}(1-\alpha)^{2} A^{2}} \geqslant 0 \\
& x_{2}^{H}=\frac{A(1-\alpha)\left[b \epsilon\left(1-\tau_{2}-2 \tau_{1}\right)-\delta\left(1-\tau_{2}+2 b \tau_{1}\right)\right]+\sqrt{\Delta(\delta)}}{2 b(1+b) \tau_{1}^{2}(1-\alpha)^{2} A^{2}}>x_{1}^{H}
\end{aligned}
$$

Since $H(0) \leqslant 0, H(+\infty)<0$ and $H^{\prime \prime}(x)<0$, we deduce that $H(x)<0$ for all $x<x_{1}^{H}$, $H(x)>0$ for all $x_{1}^{H}<x<x_{2}^{H}$ and $H(x)<0$ for all $x>x_{2}^{H}$. Note also that in the particular case where $\delta=0, H(0)=x_{1}^{H}=0$.

Since $\partial \psi(x) / \delta \tau_{1}$ has the same sign than $H(x)$, the lemma is proved.

\section{E Proof of Proposition 4}

To show Proposition 4.1 is obvious when $\delta>\delta_{H}$ (see Lemma 2). Focusing now on the configuration where $0 \leqslant \delta<\delta_{H}$, we will show that $x_{2}^{H}<x_{2}$. If this is satisfied, the result may be deduced from Lemma 2 .

To prove that $x_{2}^{H}<x_{2}$, we first note that using (A.7), $x_{2}^{H}<x^{H}$, with:

$$
x^{H} \equiv \frac{b \epsilon\left(1-\tau_{2}-2 \tau_{1}\right)-\delta\left(1-\tau_{2}+2 b \tau_{1}\right)}{b(1+b) \tau_{1}^{2}(1-\alpha) A}
$$

Substituting $x^{H}$ in $\theta(x)$ (see equation (A.1)), $\theta\left(x^{H}\right)<0$ is equivalent to:

$$
\begin{aligned}
& A \frac{\left(1-\tau_{1}-\tau_{2}\right)(1-\alpha)}{(1+b) \tau_{1}}\left[b \epsilon\left(1-\tau_{2}-2 \tau_{1}\right)-\delta\left(1-\tau_{2}-(1-b) \tau_{1}\right)\right]>(1-m)(\epsilon+\delta) \\
& +\frac{b \epsilon\left(1-\tau_{2}-2 \tau_{1}\right)-\delta\left(1-\tau_{2}+2 b \tau_{1}\right)}{b^{2}(1+b) \tau_{1}^{3}(1-\alpha)}\left[( a _ { 1 } - a _ { 2 } \tau _ { 2 } ( 1 - \alpha ) ) \left(b \epsilon\left(1-\tau_{1}-\tau_{2}\right)\right.\right. \\
& \left.\left.+\delta\left(1-\tau_{2}+b \tau_{1}\right)\right)+b \tau_{1}^{2}(1-m)(1+b)(1-\alpha)\right]
\end{aligned}
$$


This inequality is satisfied for $A$ sufficiently large. Since $A_{0}(\delta)$ is decreasing in $\delta$ and tends to $+\infty$ when $\delta$ tends to 0 , this condition is compatible with the multiplicity of steady states if $\delta$ is sufficiently low. In this case, $x_{2}^{H}$ is lower than $x_{2}$.

To prove Proposition 4.2, note that when $\delta=0, x_{1}^{H}=0$ and $x_{2}^{H}=x^{H}$. In this case, we have, of course, $x_{1}<x^{H}$ and $\partial \psi\left(x_{1}\right) / \partial \tau_{1}>0$ (see Lemma 2). By continuity, this result still holds for $\delta$ sufficiently low.

\section{F Proof of Proposition 5}

Recall that $g_{k}$ may be defined by (see equation (25)):

$$
g_{k}=1-m+\left(a_{1}-a_{2} \tau_{2}(1-\alpha)\right) A x
$$

Since $g_{k}$ is increasing in $x$ and $x$ is decreasing in $\tau_{1}$ at a stable steady state (see Proposition 4 ), we immediately deduce that $g_{k}$ is decreasing in $\tau_{1}$.

In contrast, the impact of $\tau_{2}$ on $g_{k}$ goes through two effects. Indeed, we have:

$$
\frac{d g_{k}}{d \tau_{2}}=\frac{\partial g_{k}}{\partial \tau_{2}}+\frac{\partial g_{k}}{\partial x} \frac{\partial x}{\partial \tau_{2}}
$$

with:

$$
\begin{aligned}
& \frac{\partial g_{k}}{\partial \tau_{2}}=-a_{2}(1-\alpha) A x<0 \\
& \frac{\partial g_{k}}{\partial x}=\left[a_{1}-a_{2} \tau_{2}(1-\alpha)\right] A>0
\end{aligned}
$$

When $x$ is decreasing in $\tau_{2}$, we obviously have $d g_{k} / d \tau_{2}<0$. In contrast, when $x$ is increasing in $\tau_{2}$, we have two opposite effects. To show that $d g_{k} / d \tau_{2}<0$, we first note that using (A.1), we have:

$$
\frac{\partial x}{\partial \tau_{2}}=-\frac{\partial \theta(x) / \partial \tau_{2}}{\theta^{\prime}(x)}
$$

with

$$
\begin{aligned}
\frac{\partial \theta(x)}{\partial \tau_{2}}= & -(1+b) \tau_{1}(1-\alpha)^{2} A^{2} a_{2} x^{2}+\left[(1-\alpha)^{2} A^{2} b \tau_{1}-a_{2}(1-\alpha) A(\epsilon+\delta)\right] x+\delta(1-\alpha) A \\
\theta^{\prime}(x)= & 2(1+b) \tau_{1}(1-\alpha) A^{2}\left(a_{1}-a_{2} \tau_{2}(1-\alpha)\right) x+(1-m)(1+b) \tau_{1}(1-\alpha) A \\
& +\left(a_{1}-a_{2} \tau_{2}(1-\alpha)\right) A(\epsilon+\delta)-\left(1-\tau_{1}-\tau_{2}\right)(1-\alpha)^{2} A^{2} b \tau_{1}
\end{aligned}
$$

Note that since we focus on stable steady state, we have $\theta^{\prime}(x)>0$ (see the proof of Proposition 1). Using these expressions, $d g_{k} / d \tau_{2}<0$ is equivalent to:

$$
\begin{aligned}
& (1+b) \tau_{1}(1-\alpha)^{2} A^{3} a_{2}\left(a_{1}-a_{2} \tau_{2}(1-\alpha)\right) x^{2}+\left[(1-m)(1+b) \tau_{1} a_{2}(1-\alpha)^{2} A\right. \\
& \left.+(1-\alpha)^{2} A^{2} b \tau_{1}\left(a_{1}-(1-\alpha) a_{2}\left(1-\tau_{1}\right)\right)\right] A x+\left(a_{1}-a_{2} \tau_{2}(1-\alpha)\right) A^{2}(1-\alpha) \delta>0
\end{aligned}
$$

Using $\theta(x)=0$ (see equation (A.1), we substitute $x^{2}$ in this last inequality. After some computations, we get:

$$
\begin{aligned}
& \left(a_{1}-(1-\alpha) a_{2} \tau_{2}\right)(1-\alpha) A^{2}\left[(1-\alpha) A b \tau_{1}-a_{2}(\epsilon+\delta)\right] x+A^{2}(1-\alpha) \delta\left(a_{1}-a_{2} \tau_{2}(1-\alpha)\right) \\
& +(1-\alpha)^{2} A a_{2} \delta\left(1-\tau_{1}-\tau_{2}\right)\left(A-A_{0}(\delta)\right)>0
\end{aligned}
$$


This inequality is satisfied for $A$ sufficiently close to $A_{0}(\delta)$ and $A$ large enough, such that

$A>\frac{a_{2}(\epsilon+\delta)}{(1-\alpha) b \tau_{1}}$. Note that this last condition is compatible with $A<A_{0}(\delta)$ for $\delta$ sufficiently low. This concludes the proof of the proposition.

\section{References}

[1] Balestra, C. and D. Dottori (2012) "Aging society, health and the environment", Journal of Population Economics 25, 1045-1076.

[2] Bates, D.V. (1995) "The effects of air pollution on children", Environmental Health Perspectives 103, 49-53.

[3] Blackburn, K. and Cipriani, G.P. (2002) "A model of longevity, fertility and growth", Journal of Economic Dynamics and Control 26, 187-204.

[4] Bovenberg, A.L. and S.A. Smulders (1996) "Transitional impacts of environmental policy in an endogenous growth model", International Economic Review 37, 861-893.

[5] Castelló-Climent, A. and R. Doménech (2006) "Human capital inequality, life expectancy and economic growth", Economic Journal 118, 653-677.

[6] Cervellati, M. and Sunde, U. (2005) "Human capital formation, life expectancy and the process of development", American Economic Review 95, 1653-1672.

[7] Chakraborty, S. (2004) "Endogenous lifetime and economic growth", Journal of Economic Theory 116, 119-137.

[8] Chay, K.Y. and Greenstone, M. (2003) "The impact of air pollution on infant mortality: evidence from geographic variation in pollution shocks induced by a recession", the Quarterly Journal of Economics 118, 1121-1167.

[9] Dasgupta, P. (2004) "Valuing health damages from water pollution in urban Delhi, India: a health production function approach", Environment and Development Economics 1, 83-106.

[10] Economides, G. and Philippopoulos, A. (2008) "Growth enhancing policy is a means to sustain the environment", Review of Economic Dynamics 11, 207-219.

[11] Evans, M. and Smith, K. (2005) "Do new health conditions support mortality-air pollution effects? ", Journal of Environmental Economics and Management 50, 496-518.

[12] Grossman, G. and Krueger, A. (1995) "Economic growth and the environment", Quarterly Journal of Economics 110, 353-377.

[13] Gwynn, R.C. and Thurston, G.D. (2001) "The burden of air pollution: Impacts among racial minorities", Environmental Health Perspectives 109, 501-506.

[14] Jouvet, P.-A., P. Pestieau and G. Ponthiere (2010), "Longevity and Environmental Quality in an OLG Model", Journal of Economics 100, 191-216. 
[15] Mirowsky, J. and Ross, C.E. (1998) "Education, personal control, lifestyle and health- a human capital hypothesis", Research on Ageing 20, 415-449.

[16] Neidell, M.J. (2004) "Air pollution, health and socio-economic status: the effect of outdoor air quality on childhood asthma", Journal of Health Economics 23, 1209-1236.

[17] Ono, T. (2003) "Environmental tax policy and long-run economic growth", Japanse Economic Review 54, 203-217.

[18] Palivos, T. and D. Varvarigos (2012) "Pollution abatement as a source of stabilisation and long-run growth", miméo.

[19] Pope, C.A. (2000) "Epidemiology of fine particulate air pollution and human health: biologic mechanisms and who's at risk?", Environmental Health Perspectives 108, 713723.

[20] Varvarigos, D. (2009) "Environmental degradation, longevity and the dynamics of economic development", Environmental Resources Economics 46, 59-73.

[21] World Heath Organization (2004) "Study on environmental burden of disease in children: key findings", Working Paper no EURO/05/04.

[22] World Health Organization (2006) "Health Risks of Particulate matter from long-range transboundary air pollution", Working Paper no E88189. 